\title{
Generic loss minimization for nonlinear synchronous machines by analytical computation of optimal reference currents considering copper and iron losses
}

\author{
Christoph Hackl, Julian Kullick and Niklas Monzen \\ Laboratory for Mechatronic and Renewable Energy Systems (LMRES) \\ Munich University of Applied Sciences (MUAS) \\ Munich, Germany \\ \{christoph.hackl, julian.kullick, niklas.monzen\}@ @m.edu
}

\begin{abstract}
The unified theory (introduced in [1]), which allows to analytically solve the optimal feedforward torque control (OFTC) problem of anisotropic synchronous machines (SM), is extended by considering all relevant machine nonlinearities and copper and iron losses and, thus, minimizing the overall (steady-state) losses in the machine. Instead of the well known maximum torque per current (MTPC) operation strategy, maximum torque per losses (MTPL) is realized. The unified theory for the derivation of the analytical solution is briefly recapitulated. Moreover, current and speed dependent iron losses, as well as, magnetic saturation and cross-coupling effects are considered. The resulting nonlinear optimization problem is solved via online linearization of the relevant expressions. The linearization is exemplified for flux linkages and machine torque. The presented decision tree guarantees an optimal operation management and smooth transitions between all operation strategies such as MTPL, field weakening (FW), maximum current (MC) and maximum torque per voltage (MTPV). Finally, the extended unified theory is validated for a real, highly nonlinear SM.

Index Terms-maximum torque per losses (MTPL), maximum torque per voltage (MTPV), maximum current (MC), field weakening (FW), analytical solution, efficiency, copper \& iron losses, anisotropy, synchronous machine, quadrics, quartics, Lagrangian optimisation, operation management
\end{abstract}

Notation $\mathbb{N}, \mathbb{R}$ : natural, real numbers. $\boldsymbol{x}:=$ $\left(x_{1}, \ldots, x_{n}\right)^{\top} \in \mathbb{R}^{n}$ : column vector, $n \in \mathbb{N}$ where “Т", and ":=" mean "transposed" and "is defined as", respectively. $\boldsymbol{a}^{\top} \boldsymbol{b}:=a_{1} b_{1}+\cdots+a_{n} b_{n}$ : scalar product of vectors $\boldsymbol{a}$ and $\boldsymbol{b} .\|\boldsymbol{x}\|:=\sqrt{\boldsymbol{x}^{\top} \boldsymbol{x}}=\sqrt{x_{1}^{2}+\cdots+x_{n}^{2}}$ : Euclidean norm of $\boldsymbol{x} . \boldsymbol{X} \in \mathbb{R}^{n \times m}$ : matrix with $n$ rows and $m$ columns. $\boldsymbol{I}_{n} \in \mathbb{R}^{n \times n}:=\operatorname{diag}(1, \ldots, 1)$ : identity matrix. $\boldsymbol{O}_{n \times m} \in \mathbb{R}^{n \times m}$ : zero matrix. $\mathbf{0}_{n} \in \mathbb{R}^{n}$ : zero vector. $\boldsymbol{T}_{\mathrm{p}}\left(\phi_{\mathrm{p}}\right)=\left[\begin{array}{cc}\cos \left(\phi_{\mathrm{p}}\right) & -\sin \left(\phi_{\mathrm{p}}\right) \\ \sin \left(\phi_{\mathrm{p}}\right) & \cos \left(\phi_{\mathrm{p}}\right)\end{array}\right]$ : Park transformation matrix with angle $\phi_{\mathrm{p}} \in \mathbb{R}$ and $\boldsymbol{J}:=\boldsymbol{T}_{\mathrm{p}}(\pi / 2)=\left[\begin{array}{cc}0 & -1 \\ 1 & 0\end{array}\right]$ : counter-clock wise rotation matrix (by $\frac{\pi}{2}$ ). "s.t.": subject to (optimization with constraints). $\mathbb{X} \cap \mathbb{Y}$ : intersection of sets $\mathbb{X}, \mathbb{Y} \subset \mathbb{R}^{2}$.

\section{INTRODUCTION}

In the pursuit of reducing the global energy demand, maximizing the efficiency of electric machines and drives is of paramount importance, as more than half of today's generated electricity is consumed by electric machinery [2]. In particular for synchronous machines (SMs) with anisotropic rotor designs, e.g. interior permanent magnet synchronous machines (IPMSMs), reluctance synchronous machines (RSMs) or PMassisted RMSs (PMA-RSMs), efficiency can be increased by optimal feedforward torque control (OFTC) [3, 4].

The main idea of OFTC is to exploit the ambiguity in the selection of the stator current's direct and quadrature components producing the same amount of torque, such that losses are minimized while physical constraints are satisfied (e.g. current or voltage limits). Depending on the actual operating conditions, different optimization problems may be formulated leading to respective operation strategies, such as maximum torque per current (MTPC, also known as MTPA), maximum torque per voltage (MTPV), maximum current (MC) or field weakening $(\mathrm{FW})$. Solutions to those optimization problems may be found either analytically or numerically.

Until recently, publications providing analytical solutions either neglected resistive components or assumed magnetic linearity (or both) in the problem formulation [5-8]. These unnecessary simplifications were overcome in [1] (and partly in [9-11]), where a unified theory for OFTC was proposed, offering analytical solutions for all aforementioned operation strategies while considering stator resistance and magnetic nonlinearity to minimize copper losses (only). The main idea was to reformulate the optimization problems implicitly using quadrics and finding the optimal solution by intersecting two respective quadrics. The intersection point(s) of any two quadrics can be found analytically by solving a fourth-order polynomial (quartic equation). In a more recent study [12], the solution of the intersection problem, as formulated in [1], was found numerically offering a slightly faster solution. The results, however, depend on the implementation of the root finding algorithm. In [13], an efficient Newton-Raphson-based search algorithm was used to find the optimal solutions as proposed in [1] online. Other approaches for MTPA(=MTPC in this paper) control of IPMSMs have been developed lately, e.g. based on virtual signal injection control [14], artificial neural networks [15] or a variable-equivalent-parameter MTPA control law [16]. In these publications, magnetic nonlinearity is accounted for by considering the current dependency of inductances and flux linkages. 
However, what all previous publications (including [1]) lack, though, is the consideration of iron losses which leads to suboptimal solutions (in terms of overall loss minimization), in particular at higher speeds. Numeral methods considering both copper \& iron losses and magnetic saturation usually invoke LUT-based approaches. For example, in [17] an experimental method is presented, whereas in [18] data from a finite element analysis (FEA) is used. To the best knowledge of the authors, an analytic solution of the OFTC problem considering copper $\&$ iron losses and arbitrary magnetic nonlinearity has not been published before.

\section{A. Contributions of this paper}

In this paper, the unified theory presented in [1] is extended as follows: (i) Besides copper losses, now, iron losses are considered as well in the optimization problem using a nonlinear transformer/machine model and a nonlinear (current and frequency dependent) iron resistance, (ii) the analytically obtained optimal operation strategy, i.e. maximum torque per losses (MTPL), minimizes not only ohmic losses in the stator resistance but also iron (core) losses in the stator laminations; (iii) the operation management, which determines the optimal operation strategy, is adjusted: The novel decision tree covers not only MTPL but now also allows for an analytical computation of all transition conditions (instead of solving a 16-th order polynomial numerically) and guarantees a smooth and exact transition between MPTL, MC, FW and MTPV; (iv) machine nonlinearities (e.g. due to current/speed dependencies or magnetic saturation and cross-coupling) are accounted for by online linearization and (v) the theoretical results are validated in simulations for a real, nonlinear IPMSM with current dependent stator flux linkages and differential inductances, and current/frequency dependent iron resistance.

\section{Modeling AND PROBlem STATEMEnT}

First, the nonlinear transformer/machine model, considering magnetic saturation and cross-coupling effects, as well as current and frequency dependent iron losses is presented. Then, the constrained optimization problem is stated.

\section{A. Nonlinear transformer/machine model}

The nonlinear transformer/machine model in the rotating $(d, q)$-reference frame is derived from the equivalent circuit in Fig. 1: Similar to a transformer, the stator windings are coupled with the stator iron loss circuit by the current and speed dependent stator flux linkages $\boldsymbol{\psi}_{\mathrm{s}}^{d q}=\boldsymbol{\psi}_{\mathrm{s}}^{d q}\left(\boldsymbol{i}_{\mathrm{s}}^{d q}, \omega_{\mathrm{p}}\right)$. The corresponding voltage equations of stator windings and iron core, respectively, are given by

$$
\left.\begin{array}{rl}
\boldsymbol{u}_{\mathrm{s}}^{d q} & =\boldsymbol{R}_{\mathrm{s}, \mathrm{Cu}}^{d q} \boldsymbol{i}_{\mathrm{s}}^{d q}+\omega_{\mathrm{p}} \boldsymbol{J} \boldsymbol{\psi}_{\mathrm{s}}^{d q}+\frac{\mathrm{d}}{\mathrm{d} t} \boldsymbol{\psi}_{\mathrm{s}}^{d q}, \\
\mathbf{0}_{2} & =\boldsymbol{R}_{\mathrm{s}, \mathrm{Fe}}^{d q} \boldsymbol{i}_{\mathrm{s}, \mathrm{Fe}}^{d q}+\omega_{\mathrm{p}} \boldsymbol{J} \boldsymbol{\psi}_{\mathrm{s}}^{d q}+\frac{\mathrm{d}}{\mathrm{d} t} \boldsymbol{\psi}_{\mathrm{s}}^{d q},
\end{array}\right\}
$$

with stator voltages $\boldsymbol{u}_{\mathrm{s}}^{d q}:=\left(u_{\mathrm{s}}^{d}, u_{\mathrm{s}}^{q}\right)^{\top}$, stator currents $\boldsymbol{i}_{\mathrm{s}}^{d q}:=$ $\left(i_{\mathrm{s}}^{d}, i_{\mathrm{s}}^{q}\right)^{\top}$, iron currents $i_{\mathrm{s}, \mathrm{Fe}}^{d q}:=\left(i_{\mathrm{s}, \mathrm{Fe}}^{d}, i_{\mathrm{s}, \mathrm{Fe}}^{q}\right)^{\top}$, and electrical speed $\omega_{\mathrm{p}}=n_{\mathrm{p}} \omega_{\mathrm{m}}$ (i.e. pole pair number $n_{\mathrm{p}}$ times mechanical angular velocity $\omega_{\mathrm{m}}$ ). Moreover, $\boldsymbol{R}_{\mathrm{s}, \mathrm{Cu}}^{d q}$ denotes the matrix

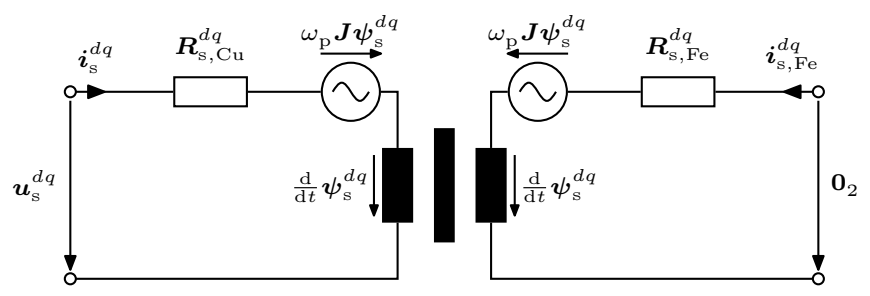

Figure 1: Equivalent circuit of the stator windings and the magnetically coupled iron core in the $d q$-reference frame.

of stator winding resistances and $\boldsymbol{R}_{\mathrm{s}, \mathrm{Fe}}^{d q}=\boldsymbol{R}_{\mathrm{s}, \mathrm{Fe}}^{d q}\left(\boldsymbol{i}_{\mathrm{s}}^{d q}, \omega_{\mathrm{p}}\right)$ is the matrix of current and frequency dependent iron resistances (both $\in \mathbb{R}^{2 \times 2}$; all entries may be present). Usually, the resistances are assumed equal for each phase, such that $\boldsymbol{R}_{\mathrm{s}, \mathrm{Cu}}^{d q}=R_{\mathrm{s}} \boldsymbol{I}_{2}$ and $\boldsymbol{R}_{\mathrm{s}, \mathrm{Fe}}^{d q}\left(\boldsymbol{i}_{\mathrm{s}}^{d q}, \omega_{\mathrm{p}}\right)=R_{\mathrm{s}, \mathrm{Fe}}\left(\boldsymbol{i}_{\mathrm{s}}^{d q}, \omega_{\mathrm{p}}\right) \boldsymbol{I}_{2}$ hold. Fig. 2 illustrates the current dependent iron losses and the corresponding iron resistance of a real IPMSM as functions of the stator currents at rated speed $\omega_{\mathrm{m}, \mathrm{R}}$. The nonlinear machine torque $m_{\mathrm{m}}$ is given by (derivation details omitted, see [19])

$$
\begin{aligned}
m_{\mathrm{m}} & =\frac{p_{\mathrm{m}}}{\omega_{\mathrm{m}}}=\frac{2 n_{\mathrm{p}}}{3 \kappa^{2}}\left(\boldsymbol{i}_{\mathrm{s}}^{d q}+\boldsymbol{i}_{\mathrm{s}, \mathrm{Fe}}^{d q}\right)^{\top} \boldsymbol{J} \boldsymbol{\psi}_{\mathrm{s}}^{d q} \\
& =\frac{2 n_{\mathrm{p}}}{3 \kappa^{2}}\left(\boldsymbol{i}_{\mathrm{s}}^{d q}-\omega_{\mathrm{p}}\left(\boldsymbol{R}_{\mathrm{s}, \mathrm{Fe}}^{d q}\right)^{-1} \boldsymbol{J} \boldsymbol{\psi}_{\mathrm{s}}^{d q}\right)^{\top} \boldsymbol{J} \boldsymbol{\psi}_{\mathrm{s}}^{d q},
\end{aligned}
$$

where $p_{\mathrm{m}}$ is the mechanical power and $\kappa \in\left\{\frac{2}{3}, \sqrt{\frac{2}{3}}\right\}$ for amplitude or power invariant Clarke transformation, respectively. The second equation is obtained by solving (1) for $\boldsymbol{i}_{\mathrm{s}, \mathrm{Fe}}^{d q}$ and inserting the (steady-state) result into (2). Note that the sum $i_{\mathrm{s}}^{d q}+\boldsymbol{i}_{\mathrm{s}, \mathrm{Fe}}^{d q}$ can be interpreted as magnetizing current.

Remark 1. The presented model is based on two simplifying (but realistic) assumptions: (A1) As only the iron power loss can be measured (not iron currents or induced voltages in iron core), the transmission ratio between stator and iron core can be chosen arbitrarily. Hence, it is reasonable to use identical flux linkages and induced voltages in stator and iron core, i.e. $\omega_{\mathrm{p}} \boldsymbol{J} \boldsymbol{\psi}_{\mathrm{s}}^{d q}+\frac{\mathrm{d}}{\mathrm{d} t} \boldsymbol{\psi}_{\mathrm{s}}^{d q}$ in (1); and (A2) the influence of iron currents on the stator flux linkages is negligible, i.e. $\psi_{\mathrm{s}}^{d q}=$ $\boldsymbol{\psi}_{\mathrm{s}}^{d q}\left(\boldsymbol{i}_{\mathrm{s}}^{d q}, \omega_{\mathrm{p}}\right)$ only depends on $\boldsymbol{i}_{\mathrm{s}}^{d q}$ and $\omega_{\mathrm{p}}$ (and not on $\left.\boldsymbol{i}_{\mathrm{s}, \mathrm{Fe}}\right)$.
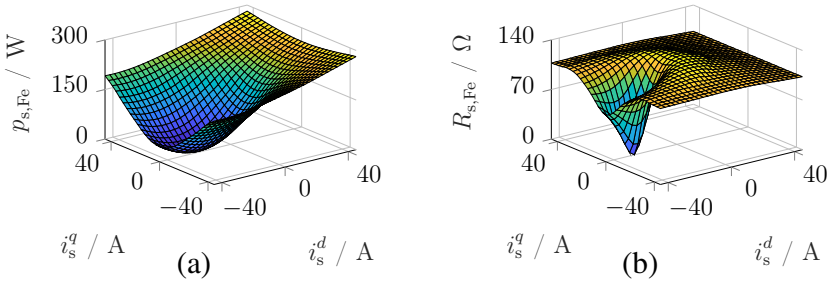

Figure 2: (a) Iron losses and (b) iron resistance at $\omega_{\mathrm{m}}=\omega_{\mathrm{m}, \mathrm{R}}$.

\section{B. Problem statement}

Main goal of OFTC is to find optimal reference currents

$\boldsymbol{i}_{\mathrm{s}, \mathrm{ref}}^{d q}\left(m_{\mathrm{m}, \mathrm{ref}}, \widehat{u}_{\mathrm{max}}, \widehat{\imath}_{\mathrm{max}}, \omega_{\mathrm{p}}\right)=\left(\begin{array}{l}i_{\mathrm{s}, \mathrm{ref}}^{d}\left(m_{\mathrm{m}, \mathrm{ref}}, \widehat{u}_{\mathrm{max}}, \widehat{\imath}_{\mathrm{max}}, \omega_{\mathrm{p}}\right) \\ i_{\mathrm{s}, \mathrm{ref}}^{q}\left(m_{\mathrm{m}, \mathrm{ref}}, \widehat{u}_{\mathrm{max}}, \widehat{\imath}_{\mathrm{max}}, \omega_{\mathrm{p}}\right)\end{array}\right)$

being functions of machine constraints $\widehat{u}_{\max } \& \widehat{\imath}_{\max }$ (maximum voltage and current magnitude, resp.), reference torque 
$m_{\mathrm{m} \text {,ref }}$ and electrical frequency $\omega_{\mathrm{p}}$ (see Fig. 3). The reference currents $\boldsymbol{i}_{\mathrm{s}, \mathrm{ref}}^{d q} \in \mathbb{S}$ must be chosen from the feasible set

$$
\mathbb{S}:=\left\{\boldsymbol{i}_{\mathrm{s}}^{d q} \in \mathbb{R}^{2} \mid\left\|\boldsymbol{u}_{\mathrm{s}}^{d q}\left(\boldsymbol{i}_{\mathrm{s}}^{d q}\right)\right\| \leq \widehat{u}_{\max } \wedge\left\|\boldsymbol{i}_{\mathrm{s}}^{d q}\right\| \leq \widehat{\imath}_{\max }\right\}
$$

(considering voltage and current limits) and are said to be optimal if they solve the following optimization problem

$$
\left.\begin{array}{l}
\boldsymbol{i}_{\mathrm{s}, \mathrm{ref}}^{d q}:=\underset{\boldsymbol{i}_{\mathrm{s}}^{d_{q}} \in \mathbb{S}}{\arg \max }-f\left(\boldsymbol{i}_{\mathrm{s}}^{d q}\right) \quad \text { s.t. } \\
\quad \operatorname{sign}\left(m_{\mathrm{m}, \mathrm{ref}}\right)=\operatorname{sign}\left(m_{\mathrm{m}}\right) \wedge\left|m_{\mathrm{m}}\right| \leq\left|m_{\mathrm{m}, \mathrm{ref}}\right|,
\end{array}\right\}
$$

subject to one inequality constraints and one equality constraint. If the (desired) reference torque $m_{\mathrm{m} \text {,ref }}$ is not feasible, then at least the maximum sign-correct torque should be provided. The cost function $f\left(\boldsymbol{i}_{\mathrm{s}}^{d q}\right)$ determines the selected optimal operation strategy; e.g. $f\left(\boldsymbol{i}_{\mathrm{s}}^{d q}\right)=\left(\boldsymbol{i}_{\mathrm{s}}^{d q}\right)^{\top} \boldsymbol{i}_{\mathrm{s}}^{d q}$ for MTPC or $f\left(\boldsymbol{i}_{\mathrm{s}}^{d q}\right)=\left(\boldsymbol{i}_{\mathrm{s}}^{d q}\right)^{\top} \boldsymbol{R}_{\mathrm{s}, \mathrm{Cu}}^{d q} i_{\mathrm{s}}^{d q}+\left(i_{\mathrm{s}, \mathrm{Fe}}^{d q}\right)^{\top} \boldsymbol{R}_{\mathrm{s}, \mathrm{Fe}}^{d q} \boldsymbol{i}_{\mathrm{s}, \mathrm{Fe}}^{d q}$ for MTPL. The OFTC problem is solved for all operation strategies by optimal reference current computation (ORCC; see Fig. 3).

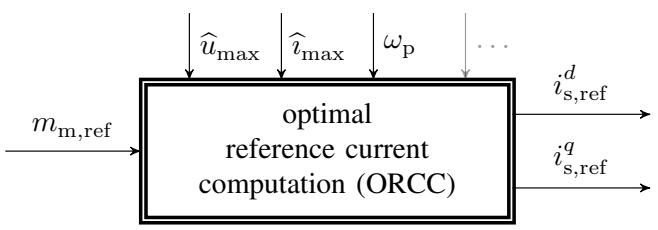

Figure 3: The optimal reference currents depend on machine constraints and actual operating condition.

Remark 2. The optimal reference currents usually also depend on stator and rotor temperature $\vartheta_{\mathrm{s}} \& \vartheta_{\mathrm{r}}$ and electrical angle $\phi_{\mathrm{p}}$ (see ... in Fig. 3; as resistances and permanent magnets are temperature dependent and slotting effects may influence the magnetic path). Due to space limitations, these are neglected here. However, the presented generic approach allows to consider these parameters in the optimization as well making huge look-up tables (LUTs) obsolete.

\section{PRoposed SOlution}

It was shown in [1] that for any operation strategy: (a) the optimization problem can be reformulated implicitely using quadrics $\left(\in \mathbb{R}^{2}\right)$ for e.g. current circle, voltage ellipse, torque hyperbola, power loss ellipse (for details see [1]), i.e.

$$
\mathbb{Q}_{\mathrm{A}}:=\{\boldsymbol{i}_{\mathrm{s}}^{d q} \in \mathbb{R}^{2} \mid \underbrace{\left(\boldsymbol{i}_{\mathrm{s}}^{d q}\right)^{\top} \boldsymbol{A} \boldsymbol{i}_{\mathrm{s}}^{d q}+2 \boldsymbol{a}^{\top} \boldsymbol{i}_{\mathrm{s}}^{d q}+\alpha}_{=: q_{\mathrm{A}}\left(\boldsymbol{i}_{\mathrm{s}}^{d q}\right)}=0\}
$$

where $\boldsymbol{A}=\boldsymbol{A}^{\top}:=\left[\begin{array}{cc}a_{11} & a_{12} \\ a_{12} & a_{22}\end{array}\right], \boldsymbol{a}:=\left(a_{1}, a_{2}\right)^{\top}$ and $\alpha \in \mathbb{R}$; (b) the optimization problem can be solved analytically by application of the Lagrangian formalism; and (c) the analytical solution (i.e. $i_{\mathrm{s}, \mathrm{ref}}^{d q}:=\left(i_{\mathrm{s}, \mathrm{ref}}^{d}, i_{\mathrm{s}, \text { ref }}^{q}\right)^{\top}$, see $\star$ in Fig. 7) is obtained by finding the optimal intersection point of two quadrics $\mathbb{Q}_{A} \cap \mathbb{Q}_{B}$ (e.g. of torque hyperbola and voltage ellipse for FW, see Fig. 7(d)).

As key theoretical aspects of the unified theory were already comprehensively covered in [1], the focus of this work is on its extension, i.e. how to (i) account for the nonlinear flux linkages $\boldsymbol{\psi}_{\mathrm{s}}^{d q}\left(\boldsymbol{i}_{\mathrm{s}}^{d q}, \omega_{\mathrm{p}}\right)$ and iron resistance $\boldsymbol{R}_{\mathrm{s}, \mathrm{Fe}}^{d q}\left(i_{\mathrm{s}}^{d q}, \omega_{\mathrm{p}}\right)$ (see online

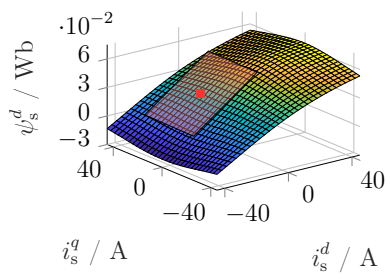

(a) $d$-flux linkage $\psi_{\mathrm{s}}^{d}\left(i_{\mathrm{s}}^{d q}\right)$.

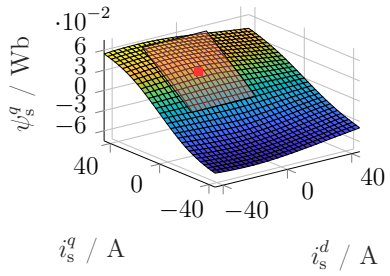

(b) $q$-flux linkage $\psi_{\mathrm{s}}^{q}\left(i_{\mathrm{s}}^{d q}\right)$.
Figure 4: Nonlinear flux linkages $\boldsymbol{\psi}_{\mathrm{s}}^{d q}\left(\boldsymbol{i}_{\mathrm{s}}^{d q}\right)$ and tangent plane $\overline{\boldsymbol{\psi}}_{\mathrm{s}}^{d q}+\overline{\boldsymbol{L}}_{\mathrm{s}}^{d q} \Delta \boldsymbol{i}_{\mathrm{s}}^{d q}[\square]$ at $\overline{\boldsymbol{i}}_{\mathrm{s}}^{d q}[\square]$ of a real anisotropic IPMSM.

linearization in Sec. III-A), (ii) define all required quadrics (see Sec. III-B), (iii) analytically compute the optimal solution of the OFTC problem considering copper and iron losses by finding the intersection point of two quadrics (see Sec. III-C) and (iv) operate the SM optimally by using a decision tree to switch between the operation strategies (see Sec. III-D).

\section{A. Online linearization}

The nonlinearity of electric machines is mainly caused by anisotropy, magnetic saturation and cross-coupling effects leading to e.g. nonlinear flux linkages $\boldsymbol{\psi}_{\mathrm{s}}^{d q}\left(\boldsymbol{i}_{\mathrm{s}}^{d q}, \omega_{\mathrm{p}}\right.$ ) (see Fig. 4), nonlinear differential inductances $\boldsymbol{L}_{\mathrm{s}}^{d q}\left(\boldsymbol{i}_{\mathrm{s}}^{d q}, \omega_{\mathrm{p}}\right)$ (see Fig. 5) and a nonlinear torque $m_{\mathrm{m}}\left(\boldsymbol{i}_{\mathrm{s}}^{d q}, \omega_{\mathrm{p}}\right)$ (see Fig. 6) which can be linearized around an operating point $\overline{\boldsymbol{x}}:=\left(\overline{\boldsymbol{i}}_{\mathrm{s}}^{d q}, \bar{\omega}_{\mathrm{p}}\right)^{\top}$. Due to space limitations, in the following, only the linearization of flux linkages and torque is presented exemplary.

Fig. 4 shows the nonlinear flux linkages of a real anisotropic IPMSM at rated speed $\omega_{\mathrm{p}}=n_{\mathrm{p}} \omega_{\mathrm{m}, \mathrm{R}}$. Clearly, the nonlinear, vector-valued flux linkages can be linearized by a first-order approximation $^{1}$ as tangent plane $[\square]$ at an arbitrary operating current $\overline{\boldsymbol{i}}_{\mathrm{s}}^{d q}[-]$ for a constant speed $\bar{\omega}_{\mathrm{p}}$ such that

$$
\boldsymbol{\psi}_{\mathrm{s}}^{d q} \approx \underbrace{\boldsymbol{\psi}_{\mathrm{s}}^{d q}\left(\overline{\boldsymbol{i}}_{\mathrm{s}}^{d q}, \bar{\omega}_{\mathrm{p}}\right)}_{=: \overline{\boldsymbol{\psi}}_{\mathrm{s}}^{d q}}+\underbrace{\left.\frac{\mathrm{d} \boldsymbol{\psi}_{\mathrm{s}}^{d q}\left(\boldsymbol{i}_{\mathrm{s}}^{d q}\right)}{\mathrm{d} \boldsymbol{i}_{\mathrm{s}}^{d q}}\right|_{\left(\overline{\boldsymbol{i}}_{\mathrm{s}}^{d q}, \bar{\omega}_{\mathrm{p}}\right)}}_{=: \overline{\boldsymbol{L}}_{\mathrm{s}}^{d q}} \cdot(\underbrace{\boldsymbol{i}_{\mathrm{s}}^{d q}-\overline{\boldsymbol{i}}_{\mathrm{s}}^{d q}}_{=: \Delta \boldsymbol{i}_{\mathrm{s}}^{d q}})
$$

holds (quantities evaluated at operating points are indicated by an overline, e.g. $\overline{\boldsymbol{\psi}}_{\mathrm{s}}^{d q}$ or $\overline{\boldsymbol{L}}_{\mathrm{s}}^{d q}$, etc.), where

$$
\overline{\boldsymbol{L}}_{\mathrm{s}}^{d q}:=\left[\begin{array}{cc}
\bar{L}_{\mathrm{s}}^{d} & \bar{L}_{\mathrm{s}, \mathrm{m}}^{d q} \\
\bar{L}_{\mathrm{s}, \mathrm{m}}^{q d} & \bar{L}_{\mathrm{s}}^{q}
\end{array}\right]:=\left.\left[\begin{array}{cc}
\frac{\partial \psi_{\mathrm{s}}^{d}}{\partial i_{\mathrm{s}}^{d}} & \frac{\partial \psi_{\mathrm{s}}^{d}}{\partial i_{\mathrm{s}}^{q}} \\
\frac{\partial \psi_{\mathrm{s}}^{q}}{\partial i_{\mathrm{s}}^{d}} & \frac{\partial \psi_{\mathrm{s}}^{q}}{\partial i_{\mathrm{s}}^{q}}
\end{array}\right]\right|_{\left(\overline{\boldsymbol{i}}_{\mathrm{s}}^{d q}, \bar{\omega}_{\mathrm{p}}\right)}=\left(\overline{\boldsymbol{L}}_{\mathrm{s}}^{d q}\right)^{\top}>0
$$

is the differential inductance matrix (evaluated at $\overline{\boldsymbol{i}}_{\mathrm{s}}^{d q}$ ). Fig. 5 shows the differential inductances and an operating point [ $\square]$.

In Fig. 6, the nonlinear torque $m_{\mathrm{m}}\left(\boldsymbol{i}_{\mathrm{s}}^{d q}, \omega_{\mathrm{p}}\right)$ of the considered real IPMSM and an exemplary reference torque $m_{\mathrm{m} \text {,ref }}$ are shown for rated speed (i.e. $\omega_{\mathrm{p}}=n_{\mathrm{p}} \omega_{\mathrm{m}, \mathrm{R}}$ ). For constant

${ }^{1}$ First-order approximation of a vector-valued function $\boldsymbol{f}(\boldsymbol{x})$ around operating point $\overline{\boldsymbol{x}}$ is given by $\boldsymbol{f}(\boldsymbol{x}) \approx \boldsymbol{f}(\overline{\boldsymbol{x}})+\left.\frac{\mathrm{d} \boldsymbol{f}(\boldsymbol{x})}{\mathrm{d} \boldsymbol{x}^{\top}}\right|_{(\overline{\boldsymbol{x}})}(\boldsymbol{x}-\overline{\boldsymbol{x}})$ (for details see [19]); e.g. for this paper, we have $\overline{\boldsymbol{x}}:=\left(\left(\overline{\boldsymbol{i}}_{\mathrm{s}}^{d q}\right)^{\top}, \bar{\omega}_{\mathrm{p}}\right)^{\top}$ neglecting $\vartheta_{\mathrm{s}}$, $\vartheta_{\mathrm{r}}$ and $\phi_{\mathrm{p}}$ (recall Remark 2). 

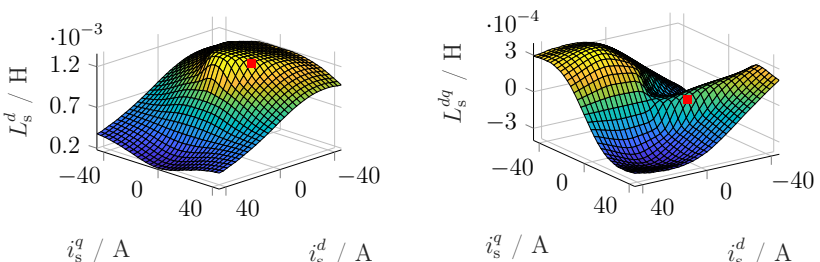

(a) $L_{\mathrm{s}}^{d}:=\frac{\partial \psi_{\mathrm{s}}^{d}}{\partial i_{\mathrm{s}}^{d}}$

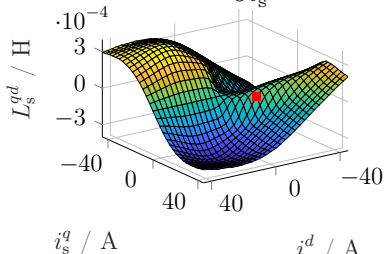

$i_{\mathrm{s}}^{q} / \mathrm{A}$

$i_{\mathrm{s}}^{d} / \mathrm{A}$

(b) $L_{\mathrm{s}, \mathrm{m}}^{d q}:=\frac{\partial \psi_{\mathrm{s}}^{d}}{\partial i_{\mathrm{s}}^{q}}$.

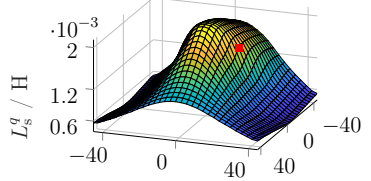

$i_{\mathrm{s}}^{q} / \mathrm{A}$

$i_{\mathrm{s}}^{d} / \mathrm{A}$

(c) $L_{\mathrm{s}, \mathrm{m}}^{q d}:=\frac{\partial \psi_{\mathrm{s}}^{q}}{\partial i_{\mathrm{s}}^{d}}$.

(d) $L_{\mathrm{s}}^{q}:=\frac{\partial \psi_{\mathrm{s}}^{q}}{\partial i_{\mathrm{s}}^{q}}$.

Figure 5: Nonlinear differential inductances $\boldsymbol{L}_{\mathrm{s}}^{d q}\left(\boldsymbol{i}_{\mathrm{s}}^{d q}\right)$ of a real anisotropic IPMSM with some operating point $\overline{\boldsymbol{i}}_{\mathrm{s}}^{d q}[\mathrm{\square}]$.

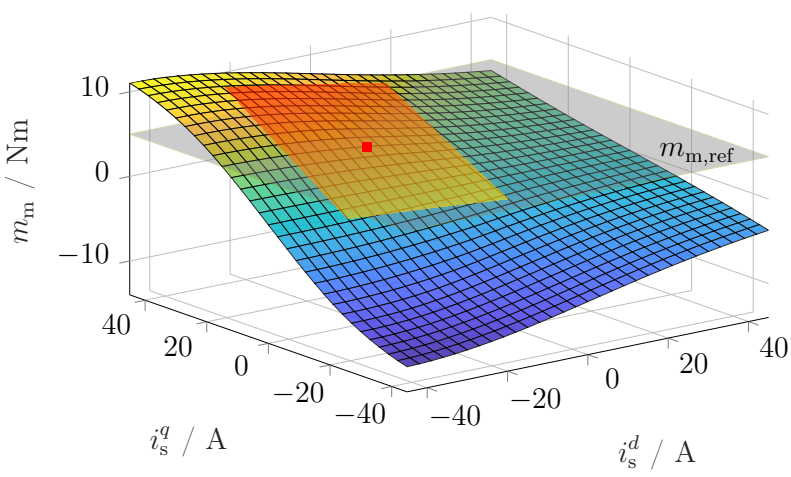

Figure 6: Nonlinear machine torque $m_{\mathrm{m}}\left(\boldsymbol{i}_{\mathrm{s}}^{d q}, \omega_{\mathrm{p}}=0\right)$ of real IPMSM with tangent surface $\left(\Delta \boldsymbol{i}_{\mathrm{s}}^{d q}\right)^{\top} \overline{\boldsymbol{T}} \Delta \boldsymbol{i}_{\mathrm{s}}^{d q}+2 \overline{\boldsymbol{t}} \Delta \boldsymbol{i}_{\mathrm{s}}^{d q}+\bar{m}_{\mathrm{m}}$ [ - ], operating point $\overline{\boldsymbol{i}}_{\mathrm{s}}^{d q}\left[{ }^{-}\right]$and reference torque $m_{\mathrm{m}, \text { ref }}[\square]$.

speed $\bar{\omega}_{\mathrm{p}}$, the illustrated tangent surface represents the more accurate second-order approximation ${ }^{2}$

$f(\boldsymbol{x}) \approx f(\overline{\boldsymbol{x}})+\left(\left.\frac{\mathrm{d} f(\boldsymbol{x})}{\mathrm{d} \boldsymbol{x}}\right|_{\overline{\boldsymbol{x}}}\right)^{\top}(\boldsymbol{x}-\overline{\boldsymbol{x}})+\frac{1}{2}(\boldsymbol{x}-\overline{\boldsymbol{x}})^{\top}\left[\left.\frac{\mathrm{d}^{2} f(\boldsymbol{x})}{\mathrm{d} \boldsymbol{x} \mathrm{d} \boldsymbol{x}^{\top}}\right|_{\overline{\boldsymbol{x}}}\right](\boldsymbol{x}-\overline{\boldsymbol{x}})$

of the torque around operating current $\overline{\boldsymbol{i}}_{\mathrm{s}}^{d q}$ [曰]. Invoking Footnote 2, the linearization of the machine torque can be

\footnotetext{
${ }^{2}$ For some $\boldsymbol{M} \in \mathbb{R}^{n \times m}, \boldsymbol{g}(\boldsymbol{x}) \in \mathbb{R}^{n}$ and $\boldsymbol{h}(\boldsymbol{x}) \in \mathbb{R}^{m}$, the second-order approximation of the scalar function $f(\boldsymbol{x}):=\boldsymbol{g}(\boldsymbol{x}) \boldsymbol{M h}(\boldsymbol{x})$ is given by (10) with gradient $\left(\frac{\mathrm{d} f(\boldsymbol{x})}{\mathrm{d} \boldsymbol{x}}\right)^{\top}=\boldsymbol{h}(\boldsymbol{x})^{\top} \boldsymbol{M}^{\top}\left[\frac{\mathrm{d} \boldsymbol{g}(\boldsymbol{x})}{\mathrm{d} \boldsymbol{x}^{\top}}\right]+\boldsymbol{g}(\boldsymbol{x})^{\top} \boldsymbol{M}\left[\frac{\mathrm{d} \boldsymbol{h}(\boldsymbol{x})}{\mathrm{d} \boldsymbol{x}^{\top}}\right]$ and symmetric Hessian matrix $\left[\frac{\mathrm{d}^{2} f(\boldsymbol{x})}{\mathrm{d} \boldsymbol{x} \mathrm{d} \boldsymbol{x}^{\top}}\right] \approx\left[\frac{\mathrm{d} \boldsymbol{h}(\boldsymbol{x})^{\top}}{\mathrm{d} \boldsymbol{x}}\right] \boldsymbol{M}^{\top}\left[\frac{\mathrm{d} \boldsymbol{g}(\boldsymbol{x})}{\mathrm{d}^{\top}}\right]+$ $\left[\frac{\mathrm{d} \boldsymbol{g}(\boldsymbol{x})^{\top}}{\mathrm{d} \boldsymbol{x}}\right] \boldsymbol{M}\left[\frac{\mathrm{d} \boldsymbol{h}(\boldsymbol{x})}{\mathrm{d} \boldsymbol{x}^{\top}}\right]$ where $\frac{\mathrm{d}}{\mathrm{d} \boldsymbol{x}}\left[\frac{\mathrm{d} \boldsymbol{g}(\boldsymbol{x})}{\mathrm{d} \boldsymbol{x}^{\top}}\right]$ and $\frac{\mathrm{d}}{\mathrm{d} \boldsymbol{x}}\left[\frac{\mathrm{d} \boldsymbol{h}(\boldsymbol{x})}{\mathrm{d}^{\top}}\right]$ are neglected (for details see [19]).
}

derived as follows

$$
m_{\mathrm{m}} \stackrel{(2)}{=} \frac{2 n_{\mathrm{p}}}{3 \kappa^{2}}(\underbrace{\boldsymbol{i}_{\mathrm{s}}^{d q}-\omega_{\mathrm{p}}\left(\boldsymbol{R}_{\mathrm{s}, \mathrm{Fe}}^{d q}\right)^{-1} \boldsymbol{J} \boldsymbol{\psi}_{\mathrm{s}}^{d q}}_{=: \boldsymbol{g}(\boldsymbol{x})})^{\top} \underbrace{\boldsymbol{I}_{2}}_{=: \boldsymbol{M}} \underbrace{\boldsymbol{J} \boldsymbol{\psi}_{\mathrm{s}}^{d q}}_{=: \boldsymbol{h}(\boldsymbol{x})}
$$

with the respective sub-derivatives (details omitted)

$$
\left.\begin{array}{l}
\left.\frac{\mathrm{d} \boldsymbol{g}(\boldsymbol{x})}{\mathrm{d} \boldsymbol{x}}\right|_{\overline{\boldsymbol{x}}}=\left.\left[\frac{\mathrm{d} \boldsymbol{i}_{\mathrm{s}}^{d q}}{\mathrm{~d} \boldsymbol{i}_{\mathrm{s}}^{d q}}-\omega_{\mathrm{p}} \frac{\mathrm{d}\left(\boldsymbol{R}_{\mathrm{s}, \mathrm{Fe}}^{d q}\right)^{-1} \boldsymbol{J} \boldsymbol{\psi}_{\mathrm{s}}^{d q}}{\mathrm{~d} \boldsymbol{i}_{\mathrm{s}}^{d q}}\right]\right|_{\left(\overline{\boldsymbol{i}}_{\mathrm{s}}^{d q}\right)}=: \boldsymbol{I}_{2}-\omega_{\mathrm{p}} \overline{\boldsymbol{Z}}_{\mathrm{i}}^{d q} \\
\quad \text { and }\left.\frac{\mathrm{d} \boldsymbol{h}(\boldsymbol{x})}{\mathrm{d} \boldsymbol{x}}\right|_{\overline{\boldsymbol{x}}}=\boldsymbol{J} \overline{\boldsymbol{L}}_{\mathrm{s}}^{d q} .
\end{array}\right\}
$$

Inserting (12) into (10) allows to rewrite the linearization of (11) as a quadratic function of the stator currents, i.e.

$$
m_{\mathrm{m}} \approx\left(\boldsymbol{i}_{\mathrm{s}}^{d q}-\overline{\boldsymbol{i}}_{\mathrm{s}}^{d q}\right)^{\top} \overline{\boldsymbol{T}}\left(\boldsymbol{i}_{\mathrm{s}}^{d q}-\overline{\boldsymbol{i}}_{\mathrm{s}}^{d q}\right)+2 \overline{\boldsymbol{t}}^{\top}\left(\boldsymbol{i}_{\mathrm{s}}^{d q}-\overline{\boldsymbol{i}}_{\mathrm{s}}^{d q}\right)+\bar{m}_{\mathrm{m}}
$$

where

$$
\begin{aligned}
\overline{\boldsymbol{T}}:= & \frac{n_{\mathrm{p}}}{3 \kappa^{2}}\left[\boldsymbol{J} \overline{\boldsymbol{L}}_{\mathrm{s}}^{d q}+\left(\boldsymbol{J} \overline{\boldsymbol{L}}_{\mathrm{s}}^{d q}\right)^{\top}\right. \\
& \left.-\omega_{\mathrm{p}}\left(\left(\overline{\boldsymbol{Z}}_{\mathrm{i}}^{d q}\right)^{\top} \boldsymbol{J} \overline{\boldsymbol{L}}_{\mathrm{s}}^{d q}+\left(\boldsymbol{J} \overline{\boldsymbol{L}}_{\mathrm{s}}^{d q}\right)^{\top} \overline{\boldsymbol{Z}}_{\mathrm{i}}^{d q}\right)\right], \\
\overline{\boldsymbol{t}}^{\top}:= & \frac{n_{\mathrm{p}}}{3 \kappa^{2}}\left(\left(\overline{\boldsymbol{i}}_{\mathrm{s}}^{d q}-\omega_{\mathrm{p}}\left(\overline{\boldsymbol{R}}_{\mathrm{s}, \mathrm{Fe}}^{d q}\right)^{-1} \overline{\boldsymbol{J}}_{\mathrm{s}}^{d q}\right)^{\top} \boldsymbol{J} \overline{\boldsymbol{L}}_{\mathrm{s}}^{d q}+\right. \\
& \left.\quad+\left(\boldsymbol{I}_{2}-\bar{\omega}_{\mathrm{p}} \overline{\boldsymbol{Z}}_{\mathrm{i}}^{d q}\right)^{\top} \boldsymbol{J} \overline{\boldsymbol{\psi}}_{\mathrm{s}}^{d q}\right) \text { and } \\
\bar{m}_{\mathrm{m}}:= & m_{\mathrm{m}}\left(\overline{\boldsymbol{i}}_{\mathrm{s}}^{d q}, \bar{\omega}_{\mathrm{p}}\right) .
\end{aligned}
$$

Besides flux linkages and machine torque, several other quantities must be linearized online; e.g. iron losses $p_{\mathrm{Fe}, \mathrm{L}}\left(\boldsymbol{i}_{\mathrm{s}}^{d q}, \omega_{\mathrm{p}}\right)=$ $\left(\boldsymbol{i}_{\mathrm{s}, \mathrm{Fe}}^{d q}\right)^{\top} \boldsymbol{R}_{\mathrm{s}, \mathrm{Fe}}^{d q} \boldsymbol{i}_{\mathrm{s}, \mathrm{Fe}}^{d q}=\omega_{\mathrm{p}}^{2}\left(\boldsymbol{\psi}_{\mathrm{s}}^{d q}\right)^{\top} \boldsymbol{J}^{\top}\left(\boldsymbol{R}_{\mathrm{s}, \mathrm{Fe}}^{d q}\right)^{-1} \boldsymbol{J} \boldsymbol{\psi}_{\mathrm{s}}^{d q}$ and voltage limit $\left\|\boldsymbol{u}_{\mathrm{s}}^{d q}\left(\boldsymbol{i}_{\mathrm{s}}^{d q}, \omega_{\mathrm{p}}\right)\right\|=u_{\max }$ need to be linearized as well with the second-order approximation introduced above (skipped due to space limitations; for details see [19]).

\section{B. Definition of machine quadrics}

After linearization, all relevant machine quadrics can be defined. For example, the linearized torque hyperbola $\overline{\mathbb{T}}:=$ $\overline{\mathbb{T}}\left(m_{\mathrm{m}, \mathrm{ref}}\right)$ describes implicitly the equality constraint (machine torque should equal reference torque, i.e. $m_{\mathrm{m}}=m_{\mathrm{m} \text {,ref }}$ ) of the optimization problem (6). One obtains

$$
\begin{aligned}
0= & m_{\mathrm{m}}-m_{\mathrm{m}, \mathrm{ref}} \\
\stackrel{(14)}{=} & \left(\boldsymbol{i}_{\mathrm{s}}^{d q}\right)^{\top} \overline{\boldsymbol{T}} \boldsymbol{i}_{\mathrm{s}}^{d q}+2 \overline{\boldsymbol{t}}^{\top} \boldsymbol{i}_{\mathrm{s}}^{d q} \\
& +\underbrace{\bar{m}_{\mathrm{m}}-2 \overline{\boldsymbol{t}} \overline{\boldsymbol{i}}_{\mathrm{s}}^{d q}+\left(\overline{\boldsymbol{i}}_{\mathrm{s}}^{d q}\right)^{\top} \overline{\boldsymbol{T}} \overline{\boldsymbol{i}}_{\mathrm{s}}^{d q}-m_{\mathrm{m}, \mathrm{ref}}}_{=: \bar{\tau}\left(m_{\mathrm{m}, \mathrm{ref}}\right)},
\end{aligned}
$$

which already has the quadratic form as in (7) and the (linearized) torque hyperbola is given by the following quadric

$$
\overline{\mathbb{T}}:=\left\{\boldsymbol{i}_{\mathrm{s}}^{d q} \in \mathbb{R}^{2} \mid\left(\boldsymbol{i}_{\mathrm{s}}^{d q}\right)^{\top} \overline{\boldsymbol{T}} \boldsymbol{i}_{\mathrm{s}}^{d q}+2 \overline{\boldsymbol{t}}^{\top} \boldsymbol{i}_{\mathrm{s}}^{d q}+\bar{\tau}=0\right\} .
$$

Another example is the current limit (circle) $\left\|\boldsymbol{i}_{\mathrm{s}}^{d q}\right\|^{2}=\widehat{\imath}_{\max }^{2}$ which can be expressed as quadric as follows

$$
\partial \mathbb{I}:=\left\{\boldsymbol{i}_{\mathrm{s}}^{d q} \in \mathbb{R}^{2} \mid\left(\boldsymbol{i}_{\mathrm{s}}^{d q}\right)^{\top} \boldsymbol{I}_{2} \boldsymbol{i}_{\mathrm{s}}^{d q}-\widehat{\imath}_{\max }^{2}=0\right\} .
$$


All other relevant (linearized) machine quadrics are summarized in Tab. I. Explicit expressions are omitted due to space limitations (for details see [1] and [19]).

Table I: Definition of (linearized) machine quadrics.

\begin{tabular}{lll}
\hline Machine quadric & $\mathbb{Q}_{\mathrm{A}}$ & $\boldsymbol{A}, \boldsymbol{a}, \alpha$ \\
\hline Current circle & $\partial \mathbb{I}\left(\widehat{\imath}_{\max }\right)$ & $\boldsymbol{I}_{2}, \mathbf{0}_{2},-\widehat{\imath}_{\max }^{2}$ \\
\hline Voltage ellipse & $\partial \overline{\mathbb{V}}\left(\widehat{u}_{\max }\right)$ & $\overline{\boldsymbol{V}}, \overline{\boldsymbol{v}}, \bar{\nu}\left(\widehat{u}_{\max }\right)$ \\
\hline Torque hyperbola & $\overline{\mathbb{T}}\left(m_{\mathrm{m}, \mathrm{ref}}\right)$ & $\overline{\boldsymbol{T}}, \overline{\boldsymbol{t}}, \bar{\tau}\left(m_{\mathrm{m}, \mathrm{ref}}\right)$ \\
\hline Power loss ellipse & $\overline{\mathbb{P L}}$ & $\overline{\boldsymbol{L}}, \overline{\boldsymbol{l}}, \bar{\rho}$ \\
\hline MTPC hyperbola & $\overline{\mathrm{M} T P \mathbb{C}}$ & $\overline{\boldsymbol{M}}_{\mathrm{C}}, \overline{\boldsymbol{m}}_{\mathrm{C}}, \bar{\mu}_{\mathrm{C}}$ \\
\hline MTPL hyperbola & $\overline{\mathbb{M} T P \mathbb{L}}$ & $\overline{\boldsymbol{M}}_{\mathrm{L}}, \overline{\boldsymbol{m}}_{\mathrm{L}}, \bar{\mu}_{\mathrm{L}}$ \\
\hline MTPV hyperbola & $\overline{\mathbb{M} T \mathbb{P V}}$ & $\overline{\boldsymbol{M}}_{\mathrm{V}}, \overline{\boldsymbol{m}}_{\mathrm{V}}, \bar{\mu}_{\mathrm{V}}$ \\
\hline
\end{tabular}

The $\operatorname{MTPX}(\mathrm{X} \in\{\mathrm{C}, \mathrm{L}, \mathrm{V}\})$ hyperbolas in Tab. I are obtained by approximating and solving the nonlinear optimization problem (6) by a quadratic programming $(Q P)$ formulation of the following form

$$
\begin{aligned}
\boldsymbol{i}_{\mathrm{s}, \text { ref }}^{d q}:=\underset{\boldsymbol{i}_{\mathrm{s}}^{d q} \in \overline{\mathbb{S}}}{\arg \max } & -\underbrace{\left(\boldsymbol{i}_{\mathrm{s}}^{d q}\right)^{\top} \boldsymbol{A} \boldsymbol{i}_{\mathrm{s}}^{d q}+2 \boldsymbol{a}^{\top} \boldsymbol{i}_{\mathrm{s}}^{d q}+\alpha}_{=: q_{\mathrm{A}}\left(\boldsymbol{i}_{\mathrm{s}}^{d q}\right)} \\
\text { s.t. } & \underbrace{\left(\boldsymbol{i}_{\mathrm{s}}^{d q}\right)^{\top} \boldsymbol{B} \boldsymbol{i}_{\mathrm{s}}^{d q}+2 \boldsymbol{b}^{\top} \boldsymbol{i}_{\mathrm{s}}^{d q}+\beta}_{=: q_{\mathrm{A}}\left(\boldsymbol{i}_{\mathrm{s}}^{d q}\right)}=0
\end{aligned}
$$

for the approximated feasible set $\overline{\mathbb{S}} \subset \mathbb{S}$ (a subset of $\mathbb{S}$ as in (5)). By applying the Lagrangian formalism to (17), an implicit solution (again a quadric) is obtained which describes the respective MTPX hyperbolas (for more details see [1]).

\section{Intersection of two quadrics}

Using the quadrics listed in Tab. I, an analytical solution for the different operation strategies can be computed by finding the optimal intersection point of two quadrics $\mathbb{Q}_{\mathrm{A}}$ and $\mathbb{Q}_{\mathrm{B}}$, i.e.

$$
\boldsymbol{i}_{\mathrm{s}, \mathrm{ref}}^{d q}=\boldsymbol{i}_{\mathrm{s}, \mathrm{X}}^{d q}:=\mathbb{Q}_{\mathrm{A}} \cap \mathbb{Q}_{\mathrm{A}}, \mathrm{X} \in\{\mathrm{MTPL}, \mathrm{MC}, \mathrm{FW}, \ldots\}
$$

(for details see Tab. II and [1]). The computation problem narrows down to the problem of finding the roots of a fourth-order polynomial; for which analytic solutions exist (e.g. Ferrari's method, see [1]). The choices of $\mathbb{Q}_{\mathrm{A}}$ and $\mathbb{Q}_{\mathrm{B}}$ depend on the relevant (feasible) operation strategy (see next section) and are listed for all operation strategies in Tab. II.

\section{Operation management (decision tree)}

First, all operation strategies, illustrated in Fig. 7, and, then, the optimal operation management, i.e. the decision tree shown in Fig. 8, are explained. To do so, it is reasonable to recall the overall goal of OFTC: Providing the desired machine torque (if not feasible, the maximum torque) according to a given reference torque in the most efficient way. In Fig. 7, the second quadrant of the current locus is plotted for different operating conditions (i.e. varying speeds $\omega_{p}$ and reference

\begin{tabular}{|c|c|}
\hline $\begin{array}{l}\text { Strategy } \\
\quad \text { Optimal reference current }\end{array}$ & $\begin{array}{l}\mathbb{Q}_{\mathrm{A}} \text { with } \boldsymbol{A}, \boldsymbol{a}, \alpha ; \\
\mathbb{Q}_{\mathrm{B}} \text { with } \boldsymbol{B}, \boldsymbol{b}, \beta\end{array}$ \\
\hline $\begin{array}{l}\text { MTPC } \\
\quad \boldsymbol{i}_{\mathrm{s}, \mathrm{MTPC}}^{d q}=\overline{\mathbb{M T} \mathbb{P} \mathbb{C}} \cap \overline{\mathbb{T}}\left(m_{\mathrm{m}, \mathrm{ref}}\right)\end{array}$ & $\begin{array}{l}\overline{\boldsymbol{M}}_{\mathrm{C}}, \overline{\boldsymbol{m}}_{\mathrm{C}}, 0 \\
\overline{\boldsymbol{T}}, \overline{\boldsymbol{t}}, \bar{\tau}\left(m_{\mathrm{m}, \mathrm{ref}}\right)\end{array}$ \\
\hline 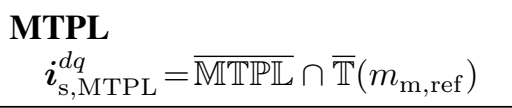 & $\begin{array}{l}\overline{\boldsymbol{M}}_{\mathrm{L}}, \overline{\boldsymbol{m}}_{\mathrm{L}}, \bar{\mu}_{\mathrm{L}} \\
\overline{\boldsymbol{T}}, \overline{\boldsymbol{t}}, \bar{\tau}\left(m_{\mathrm{m}, \mathrm{ref}}\right)\end{array}$ \\
\hline $\begin{array}{l}\mathbf{M C} \\
\qquad \boldsymbol{i}_{\mathrm{s}, \mathrm{MC}}^{d q}=\partial \mathbb{I}\left(\widehat{\imath}_{\max }\right) \cap \overline{\mathbb{V}}\left(\widehat{u}_{\max }\right)\end{array}$ & $\begin{array}{l}\boldsymbol{I}_{2}, \mathbf{0}_{2},-\widehat{\imath}_{\max }^{2} \\
\overline{\boldsymbol{V}}, \overline{\boldsymbol{v}}, \bar{\nu}\left(\widehat{u}_{\max }\right)\end{array}$ \\
\hline $\begin{array}{l}\mathbf{M C}_{\mathrm{ext}} \\
\quad \boldsymbol{i}_{\mathrm{s}, \mathrm{MC}_{\mathrm{ext}}}^{d q}=\partial \mathbb{I}\left(\widehat{\imath}_{\max }\right) \cap \overline{\mathbb{T}}\left(m_{\mathrm{m}, \mathrm{ref}}\right)\end{array}$ & $\begin{array}{l}\boldsymbol{I}_{2}, \mathbf{0}_{2},-\widehat{\imath}_{\max }^{2} \\
\overline{\boldsymbol{T}}, \overline{\boldsymbol{t}}, \bar{\tau}\left(m_{\mathrm{m}, \mathrm{ref}}\right)\end{array}$ \\
\hline $\begin{array}{l}\mathbf{F W} \\
\quad \boldsymbol{i}_{\mathrm{s}, \mathrm{FW}}^{d q}=\partial \overline{\mathbb{V}}\left(\widehat{u}_{\max }\right) \cap \overline{\mathbb{T}}\left(m_{\mathrm{m}, \mathrm{ref}}\right)\end{array}$ & $\begin{array}{l}\overline{\boldsymbol{T}}, \overline{\boldsymbol{t}}, \bar{\tau}\left(m_{\mathrm{m}, \mathrm{ref}}\right) \\
\overline{\boldsymbol{V}}, \overline{\boldsymbol{v}}, \bar{\nu}\left(\widehat{u}_{\max }\right)\end{array}$ \\
\hline $\begin{array}{l}\text { MTPV } \\
\quad \boldsymbol{i}_{\mathrm{s}, \mathrm{MTPV}}^{d q}=\overline{\mathbb{M} T \mathbb{P} \mathbb{V}} \cap \partial \overline{\mathbb{V}}\left(\widehat{u}_{\max }\right)\end{array}$ & $\begin{array}{l}\overline{\boldsymbol{M}}_{\mathrm{V}}, \overline{\boldsymbol{m}}_{\mathrm{V}}, \bar{\mu}_{\mathrm{V}} \\
\overline{\boldsymbol{V}}, \overline{\boldsymbol{v}}, \bar{\nu}\left(\widehat{u}_{\max }\right)\end{array}$ \\
\hline
\end{tabular}

Table II: Optimal reference current computation (ORCC) by intersecting two quadrics $\mathbb{Q}_{\mathrm{A}} \& \mathbb{Q}_{\mathrm{B}}$ for all operation strategies.

torques $m_{\mathrm{m}, \mathrm{ref}}$ ). In each subplot, the machine quadrics (colored lines), as well as the approximated feasible set $\overline{\mathbb{S}} \subset \mathbb{S}$ [ ] and the optimal reference currents $\boldsymbol{i}_{\mathrm{s}, \text { ref }}^{d q}[\boldsymbol{t}$ ] are plotted. The presented subplots are used to illustrate the different operation strategies.

In the low to moderate speed range and/or for small reference torques, the overall goal of OFTC can be achieved by the MTPL strategy. MTPL is defined as the intersection [ $\boldsymbol{t}$ ] of MTPL hyperbola [-] and torque hyperbola [-], i.e. $\overline{\mathrm{MTPL}} \cap \overline{\mathbb{T}}\left(m_{\mathrm{m}, \mathrm{ref}}\right)$. This condition is fulfilled in subplots (a) and (b) of Fig. 7. In contrast to the MTPC strategy-which is the intersection of MTPC hyperbola [-] and torque hyperbola $[-]$, i.e. $\overline{\mathbb{M T P C}} \cap \overline{\mathbb{T}}\left(m_{\mathrm{m}, \text { ref }}\right)$-MTPL minimizes not only copper losses but also iron losses. It can be observed in subplots (a) and (b) of Fig. 7, that the MTPL hyperbola moves to the left for increasing speeds, whereas the MTPC hyperbola remains unchanged at its initial location.

If the reference torque is too high and cannot be provided by MTPL (see subplot (c) of Fig. 7), where the intersection of $\overline{\mathrm{MTPL}} \cap \overline{\mathbb{T}}\left(m_{\mathrm{m}, \mathrm{ref}}\right)$ is located outside the current circle $[-])$, the extended maximum current $\left(\mathrm{MC}_{\mathrm{ext}}\right)$ operation strategy is opted for as here the reference torque is still feasible. It is obtained by the intersection [ $\boldsymbol{\lambda}$ ] of current circle $[-]$ and torque hyperbola $[-]$, i.e. $\partial \mathbb{I}\left(\widehat{\imath}_{\max }\right) \cap \overline{\mathbb{T}}\left(m_{\mathrm{m} \text {,ref }}\right)$. For $\mathrm{MC}_{\text {ext }}$, the objective of providing the reference torque is prioritized over the objective of minimizing losses.

If the torque is lowered, but the speed is increased, as shown in subplot (d) of Fig. 7, the field, weakening (FW) strategy becomes active, as MTPL is not feasible anymore. FW is defined as the intersection of voltage ellipse $[-]$ and torque hyperbola $[-]$, i.e. $\partial \overline{\mathbb{V}}\left(\widehat{u}_{\max }\right) \cap \overline{\mathbb{T}}\left(m_{\mathrm{m} \text {,ref }}\right)$.

If, for the same reference torque, the speed increases such that the torque hyperbola is located outside the feasible 


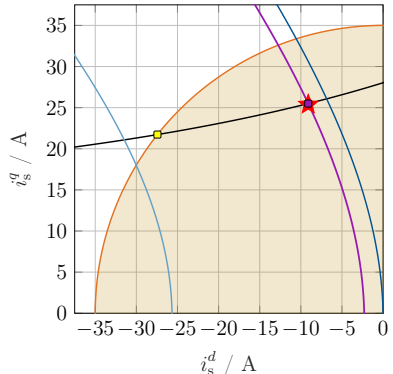

(a) MTPL $\left(\frac{1}{2} \omega_{\mathrm{p}, \mathrm{R}}, \frac{3}{4} m_{\mathrm{m}, \mathrm{R}}\right)$.

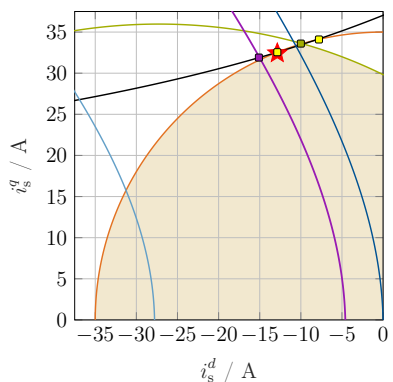

(c) $\mathrm{MC}_{\mathrm{ext}}\left(\omega_{\mathrm{p}, \mathrm{R}}, m_{\mathrm{m}, \mathrm{R}}\right)$.

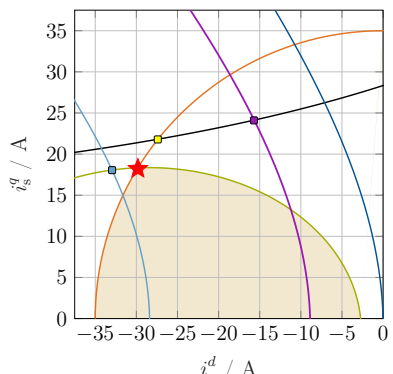

(e) $\operatorname{MC}\left(2 \omega_{\mathrm{p}, \mathrm{R}}, \frac{3}{4} m_{\mathrm{m}, \mathrm{R}}\right)$.

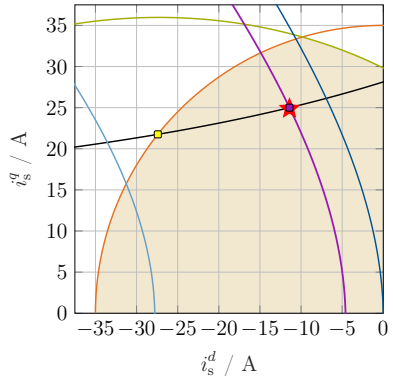

(b) $\operatorname{MTPL}\left(\omega_{\mathrm{p}, \mathrm{R}}, \frac{3}{4} m_{\mathrm{m}, \mathrm{R}}\right)$.

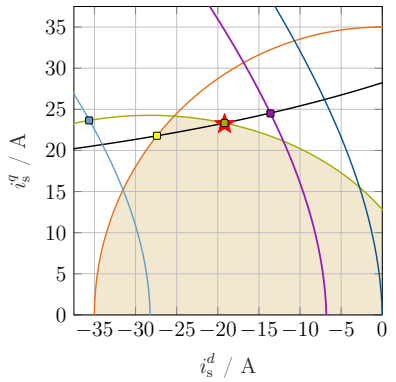

(d) FW $\left(\frac{5}{2} \omega_{\mathrm{p}, \mathrm{R}}, \frac{3}{4} m_{\mathrm{m}, \mathrm{R}}\right)$.

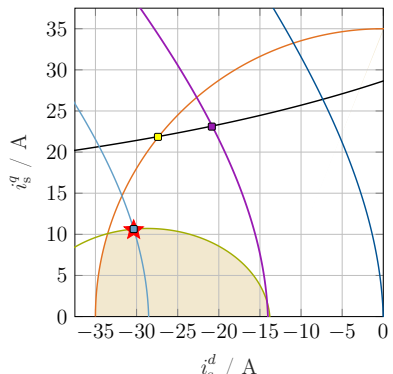

(f) $\operatorname{MTPV}\left(\frac{7}{2} \omega_{\mathrm{p}, \mathrm{R}}, \frac{3}{4} m_{\mathrm{m}, \mathrm{R}}\right)$.

Figure 7: Current locus for different operating conditions with different electrical angular frequencies and reference torques $\left(\omega_{\mathrm{p}}, m_{\mathrm{m}, \mathrm{ref}}\right)$; shown are all quadrics for $\partial \mathbb{I}\left(\widehat{\imath}_{\max }\right)[-]$, $\overline{\mathbb{T}}\left(m_{\text {m,ref }}\right)[-], \partial \overline{\mathbb{V}}\left(\widehat{u}_{\text {max }}\right)$ [-], $\overline{\mathbb{M T P C}}[-], \overline{\mathrm{MTP} P}$ $[-], \overline{\mathbb{M T P V}}[-]$, feasible region $\overline{\mathbb{S}}:=\overline{\mathbb{V}}\left(\widehat{u}_{\text {max }}\right) \cap \mathbb{I}\left(\widehat{\imath}_{\text {max }}\right)$ [ $\quad$ ] and reference currents $\boldsymbol{i}_{\mathrm{s}, \mathrm{ref}}^{d q}[\boldsymbol{\downarrow}]$.

region-see subplot (e) of Fig. 7-the maximum current (MC) strategy must be applied. For MC, which is the intersection [ $\star$ ] of current circle [-] and voltage ellipse [-], i.e. $\partial \mathbb{I}\left(\widehat{\imath}_{\max }\right) \cap \overline{\mathbb{V}}\left(\widehat{u}_{\text {max }}\right)$, the reference torque cannot be reached anymore. Instead the maximum feasible torque is provided.

Finally, if the speed increases even further and the reference torque remains the same, MTPV is selected which is illustrated in subplot (f) of Fig. 7. For MTPV, the intersection [ $\star$ ] of MTPV hyperbola [-] and voltage ellipse [-] defines the reference current vector, i.e. $\overline{\mathbb{M} T P V} \cap \partial \bar{V}\left(\widehat{u}_{\max }\right)$. MTPV is, as MC, a suboptimal solution as the reference torque is not feasible anymore; only the maximum feasible torque is provided by both operation strategies.

For operation management, in order to guarantee a smooth transition between the operation strategies, the decision tree in Fig. 8 is proposed. Initially, at each control instant, the input

variables are updated (if not constant), e.g. by measurements or from LUTs. In the next step, the quadric parameters are recomputed using the updated input variables. The reference torque (which e.g. may be the output of a speed controller) should be limited by the maximum (or minimal) feasible motor torque $m_{\mathrm{m}, \max }>0$ (or generator torque $m_{\mathrm{m}, \min }<0$ ) due to the current limit. Next, the MTPL reference currents $i_{\mathrm{s}, \mathrm{MTPL}}^{d q}$ are calculated. If located in the feasible region, i.e. $i_{\mathrm{s}, \mathrm{MTPL}}^{d q} \in \overline{\mathbb{S}}, \mathrm{MTPL}$ is selected. If not the $\mathrm{MC}_{\text {ext }}$ reference currents $i_{\mathrm{s}, \mathrm{MC}_{\text {ext }}}^{d q}$ are calculated. If the $d$-component of the MTPL reference currents is smaller than the $d$-component of the $\mathrm{MC}_{\text {ext }}$ reference currents and the $\mathrm{MC}_{\text {ext }}$ reference currents are inside the feasible region, i.e. $\boldsymbol{i}_{\mathrm{s}, \mathrm{MC}_{\mathrm{ext}}}^{d q} \in \overline{\mathbb{S}}$, the $\mathrm{MC}_{\text {ext }}$ strategy is selected. If not the FW reference currents $i_{\mathrm{s}, \mathrm{FW}}^{d q}$ are calculated. If located within the feasible region, i.e. $i_{\mathrm{s}, \mathrm{FW}}^{d q} \in \overline{\mathbb{S}}$, the FW strategy is selected. If not, in the last conditional branch, it is checked whether the MTPV reference currents $\boldsymbol{i}_{\mathrm{s}, \mathrm{MTPV}}^{d q}$ are located within the feasible region, i.e. $i_{\mathrm{s}, \mathrm{MTPV}}^{d q} \in \overline{\mathrm{S}}$. If yes, MTPV is applied. Otherwise, MC is the last remaining option.

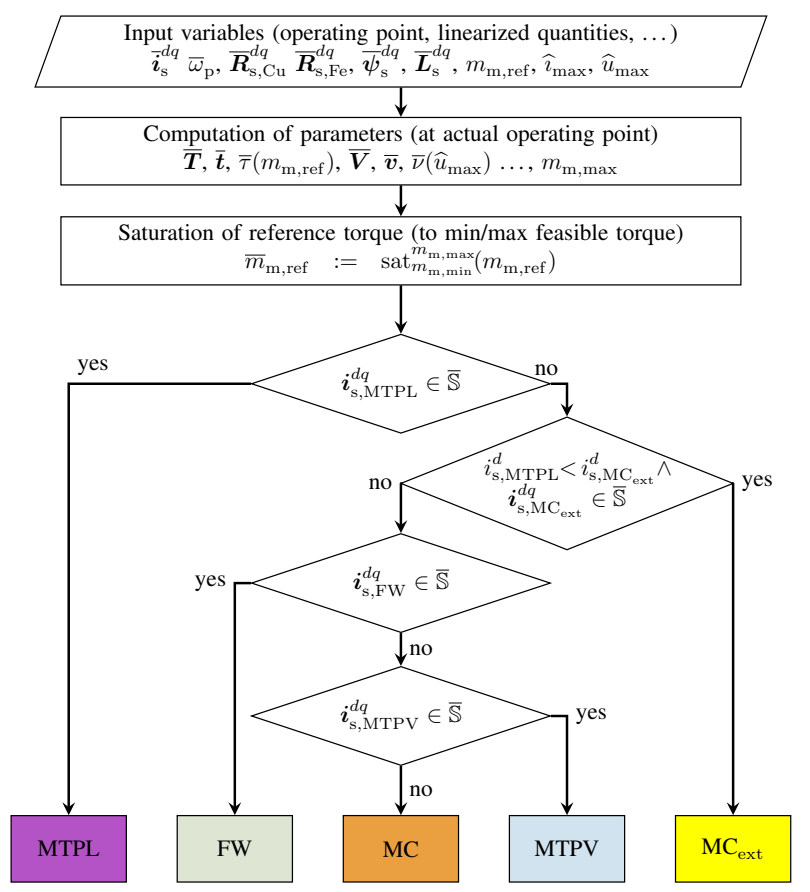

Figure 8: Decision tree for optimal operation management.

\section{Simulation RESUltS}

The analytic computation of the optimal reference currents in accordance with Tab. II and the decision tree in Fig. 8 are implemented in Matlab \& Simulink R2019b. For the simulations, the proposed optimal reference current computation (ORCC) is used for field-orientated control (FOC) of a real, anisotropic and nonlinear IPMSM having the parameters listed in Tab. III. The reference torque is provided by an outer-loop speed controller, whereas the computed reference currents are forwarded to the inner-loop stator current controllers. 
Table III: Parameters of the simulated but real anisotropic SM.

\begin{tabular}{llr}
\hline Parameter & Symbol & Value \\
\hline Rated mech. power & $p_{\mathrm{m}, \mathrm{R}}$ & $4 \mathrm{~kW}$ \\
Rated mech. speed & $n_{\mathrm{m}, \mathrm{R}}$ & $5500 \mathrm{rpm}$ \\
Rated mach. torque & $m_{\mathrm{m}, \mathrm{R}}$ & $6.6 \mathrm{~N} \mathrm{~m}$ \\
Maximum voltage & $\widehat{u}_{\mathrm{max}}$ & $135 \mathrm{~V}$ \\
Maximum current & $\widehat{\imath}_{\max }$ & $35 \mathrm{~A}$ \\
Nonlinear torque & $m_{\mathrm{m}}$ & see Fig. 6 \\
Nonlinear flux linkages & $\boldsymbol{\psi}_{\mathrm{s}}^{d q}$ & see Fig. 4 \\
Nonlinear iron losses & $p_{\mathrm{s}, \mathrm{Fe}}, R_{\mathrm{s}, \mathrm{Fe}}$ & see Fig. 2 \\
Differential inductances & $L_{\mathrm{s}}^{d}, L_{\mathrm{s}}^{q}, L_{\mathrm{s}, \mathrm{m}}$ & see Fig. 5 \\
Stator resistance & $R_{\mathrm{s}}$ & $0.2 \Omega$ \\
\hline
\end{tabular}

A start-up scenario, from stand-still to $250 \%$ of rated speed $n_{\mathrm{m}, \mathrm{R}}$, is simulated. The load torque is fixed at $50 \%$ of the rated torque $m_{\mathrm{m}, \mathrm{R}}$. The simulation illustrates the whole speed range and smooth transitions between all operating strategies such as MTPL [ ], MC ext [ ], MC [ ], MTPV [ ] and FW [ ]. Fig. 9 shows (from top to bottom), the time series of actual [-] and reference or maximum values [---] of stator currents $i_{\mathrm{s}}^{q}$ and $i_{\mathrm{s}}^{d}$, voltage magnitude $\left\|\boldsymbol{u}_{\mathrm{s}}^{d q}\right\|$, machine speed $n_{\mathrm{m}}$ and torque $m_{\mathrm{m}}$. The background colors indicate the active (optimal) operating strategy. In Fig. 10, for the same simulation, the speed-torque map with efficiency contour lines (see Fig. 10a) and the current locus (see Fig. 10b) are shown. The actual speed-torque pairs $\left(n_{\mathrm{m}}, m_{\mathrm{m}}\right)$ or currents $\left(i_{\mathrm{s}}^{d}, i_{\mathrm{s}}^{q}\right)$ are marked by [-]. The corresponding reference values are highlighted according to the assigned colors of the actual operation strategy (cf. Fig. 9). The final (optimal) operating point is marked by [ $\widehat{T}]$.

In Fig. 9, at the beginning ( $t \leq 0.1 \mathrm{~s}$ ), the machine is slowly ramped up to $20 \%$ of $n_{\mathrm{m}, \mathrm{R}}$ which is reached at $t=0.07 \mathrm{~s}$. During this period, the optimal currents $\boldsymbol{i}_{\mathrm{s}, \mathrm{MTPL}}^{d q}$ are obtained by intersecting torque and MTPL hyperbolas (i.e. $\left.\overline{\mathrm{MTPL}} \cap \overline{\mathbb{T}}\left(m_{\mathrm{m}, \text { ref }}\right)\right)$. The currents are within the feasible set (i.e. $i_{\mathrm{s}, \mathrm{MTPL}}^{d q} \in \overline{\mathbb{S}}$ ) and, therefore, the MTPL strategy [ ] is applied.

At time $t=0.1 \mathrm{~s}$, the reference speed jumps to $250 \%$ of $n_{\mathrm{m}, \mathrm{R}}$. Consequently, to accelerate further, the reference torque jumps to the maximum torque $m_{\mathrm{m} \text {,max }}$. Due to the fact that the maximum torque cannot be reached by the MTPL strategy, now, the $\mathrm{MC}_{\text {ext }}$ strategy [ ] is active until $t=0.205 \mathrm{~s}$.

For $t \in[0.205 \mathrm{~s}, 0.43 \mathrm{~s})$, current and voltage limits are reached due to increasing speed. The applied operation strategy is MC [ ]. It is used until The shrinking voltage ellipse 'pushes' the optimal reference currents onto the current circle along the negative $d$-axis. The intersection of both respective quadrics, i.e. $\partial \overline{\mathbb{V}}\left(\widehat{u}_{\max }\right) \cap \partial \mathbb{I}\left(\widehat{\imath}_{\max }\right)$, gives the optimal reference current that provides the maximum feasible torque, which is, however, smaller than the (saturated) reference torque, i.e. $m_{\mathrm{m}}<\bar{m}_{\mathrm{m}, \mathrm{ref}}=m_{\mathrm{m}, \max }$.

At time $t=0.43 \mathrm{~s}$, the voltage limit dominates and the operation management changes to the MTPV strategy [ ] to pro-

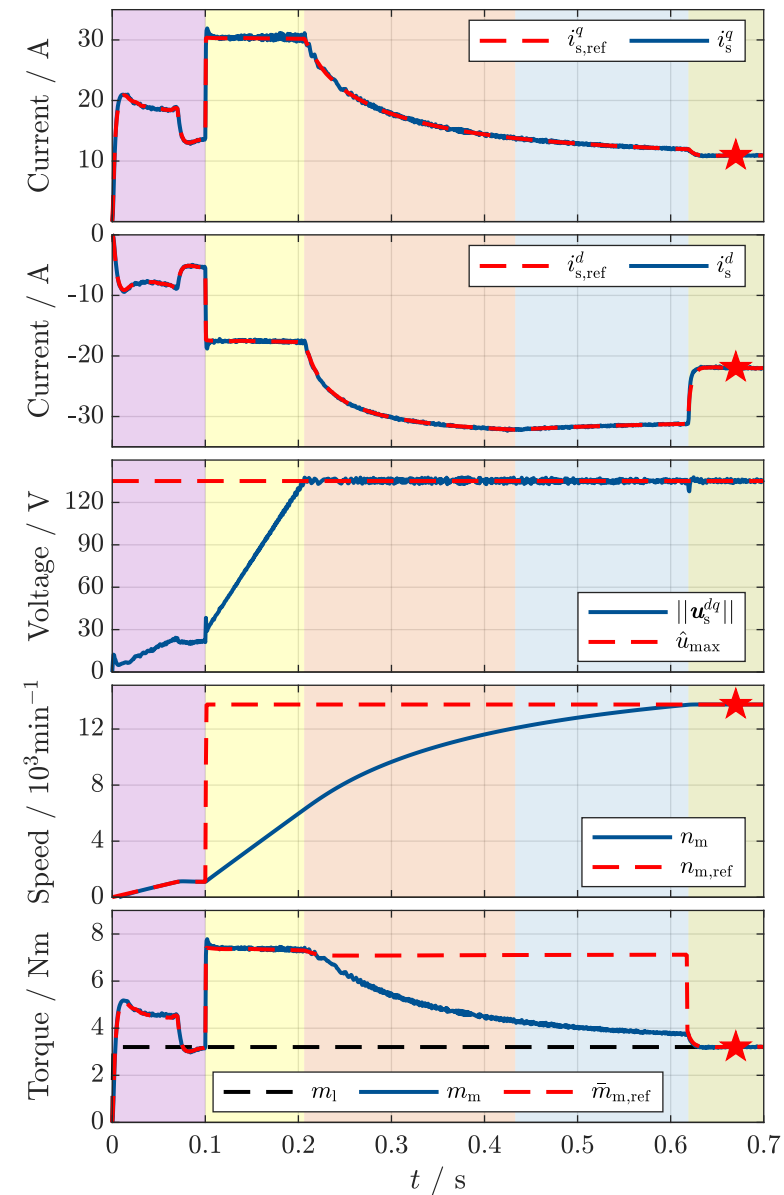

Figure 9: Simulated time series results of smooth transition between all operation strategies, i.e. MTPL [ ], $\operatorname{MC}_{\text {ext }}[\quad]$, MC [ ], MTPV [ ] and FW [ ].

vide the maximum feasible torque (but again $m_{\mathrm{m}}<\bar{m}_{\mathrm{m} \text {,ref }}$ ). The reference currents are computed as the intersection of MTPV hyperbola and voltage ellipse (i.e. $\overline{\mathbb{M} T P \mathbb{V}} \cap \partial \overline{\mathbb{V}}\left(\widehat{u}_{\max }\right)$ ).

By reaching the reference speed at $t=0.62 \mathrm{~s}$, no more acceleration is needed and the remaining reference torque must only compensate for the load torque $m_{1}=0.5 m_{\mathrm{m}, \mathrm{R}}=$ $m_{\mathrm{m} \text {,ref }}=m_{\mathrm{m}}$. As a result, finally, the FW strategy [ ] is active. The start-up scenario is completed and the speed is kept constant until the simulation ends at $t=0.7 \mathrm{~s}$.

Concluding, Fig. 11 shows additional simulation results comparing MTPL and MTPC for four operating conditions $B_{11}=\left(0.3 n_{\mathrm{m}, \mathrm{R}}, 0.3 m_{\mathrm{m}, \mathrm{R}}\right), B_{12}=\left(0.3 n_{\mathrm{m}, \mathrm{R}}, m_{\mathrm{m}, \mathrm{R}}\right), B_{21}=$ $\left(n_{\mathrm{m}, \mathrm{R}}, 0.3 m_{\mathrm{m}, \mathrm{R}}\right)$ and $B_{22}=\left(n_{\mathrm{m}, \mathrm{R}}, m_{\mathrm{m}, \mathrm{R}}\right)$ with different speed and torque pairs. It becomes clear that considering copper and iron losses leads to efficiency improvements over the whole operation range. In particular, the highest efficiency gain $(>2 \%)$ is reached at high speed and low torque (i.e. $B_{21}$ ), due to the fact, that additional negative $d$-currents decrease iron losses more than increasing copper losses. 


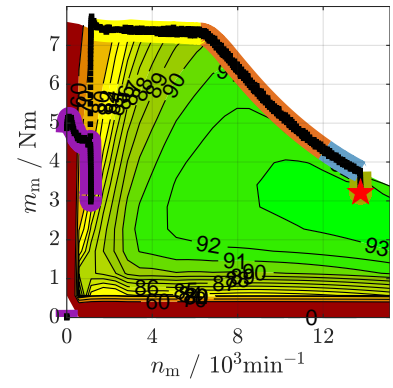

(a)

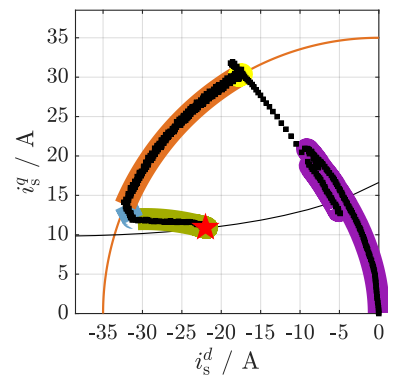

(b)
Figure 10: Illustration of smooth transition between all operation strategies: (a) speed-torque map and (b) current locus.

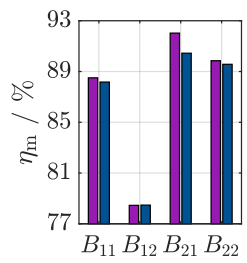

(a)

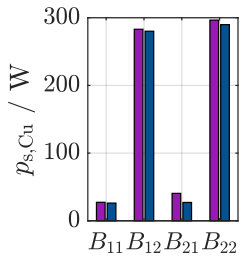

(b)

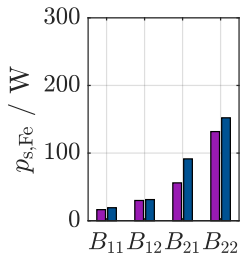

(c)
Figure 11: Comparison of MTPL [ $[$ ] and MTPC [ $]$ strategies: (a) efficiency, (b) copper losses and (c) iron losses.

\section{CONCLuSion}

A generic and analytical solution for the optimal feedforward torque control problem of nonlinear synchronous machines (SMs) was presented. The unified theory proposed in [1] was extended by incorporating current and speed dependent iron losses and accounting for nonlinear flux linkages and differential inductances by online linearization during the online optimization. In addition, a simple(r) decision tree for the operation management was presented, whose transition conditions now can be computed analytically and more easily than in [1]. The results were validated by simulations using a highly nonlinear, current/speed-dependent machine model of a real SM. It was shown, that optimal operation was guaranteed over the whole speed range and a smooth transition between all operation strategies was achieved. Future work will verify the theoretical and simulative results in the laboratory.

\section{REFERENCES}

[1] H. Eldeeb, C. M. Hackl, L. Horlbeck, and J. Kullick, “A unified theory for optimal feedforward torque control of anisotropic synchronous machines," International Journal of Control, 2017.

[2] A. De Almeida, F. Ferreira, and J. Fong, "Standards for efficiency of electric motors," IEEE Industry Applications Magazine, vol. 17, pp. 12-19, jan 2011.

[3] I. Boldea, L. Tutelea, Lucian N. Parsa, and D. Dorrell, "Automotive electric propulsion systems with reduced or no permanent magnets: An overview," IEEE Transactions on Industrial Electronics, vol. 61, pp. 5696-5711, oct 2014.

[4] E. Schmidt, "Synchronous reluctance machines with highanisotropy rotors - comparison of their operational characteristics," in 2014 Australasian Universities Power Engineering Conference (AUPEC), pp. 1-6, Institute of Electrical and Electronics Engineers (IEEE), sep 2014.
[5] M. Tursini, E. Chiricozzi, and R. Petrella, "Feedforward fluxweakening control of surface-mounted permanent-magnet synchronous motors accounting for resistive voltage drop," IEEE Transactions on Industrial Electronics, vol. 57, pp. 440-448, jan 2010.

[6] S.-Y. Jung, J. Hong, and K. Nam, "Current minimizing torque control of the IPMSM using Ferrari's method," IEEE Transactions on Power Electronics, vol. 28, pp. 5603-5617, dec 2013.

[7] M. Preindl and S. Bolognani, "Optimal state reference computation with constrained MTPA Criterion for PM motor drives," IEEE Transactions on Power Electronics, vol. 30, pp. 45244535, aug 2015.

[8] J. Lemmens, P. Vanassche, and J. Driesen, "PMSM drive current and voltage limiting as a constraint optimal control problem," IEEE Journal of Emerging and Selected Topics in Power Electronics, vol. 3, pp. 326-338, jun 2015.

[9] L. Horlbeck and C. M. Hackl, "Analytical solution for the MTPV hyperbola including the stator resistance," in Proceedings of the IEEE International Conference on Industrial Technology (ICIT 2016), (Taipei, Taiwan), pp. 1060-1067, Institute of Electrical and Electronics Engineers (IEEE), mar 2016.

[10] H. Eldeeb, C. M. Hackl, L. Horlbeck, and J. Kullick, "Analytical solutions for the optimal reference currents for MTPC/MTPA, MTPV and MTPF control of anisotropic synchronous machines," in Proceedings of the IEEE International Electric Machines \& Drives Conference (IEMDC 2017), (Miami, FL, USA), pp. 1-6, 2017.

[11] C. M. Hackl, J. Kullick, H. Eldeeb, and L. Horlbeck, "Analytical computation of the optimal reference currents for MTPC/MTPA, MTPV and MTPF operation of anisotropic synchronous machines considering stator resistance and mutual inductance," in 2017 19th European Conference on Power Electronics and Applications (EPE'17 ECCE Europe), pp. P.1-P.10, Sept 2017.

[12] K. Choi, Y. Kim, K.-S. Kim, and S.-K. Kim, "Real-time optimal torque control of interior permanent magnet synchronous motors based on a numerical optimization technique," IEEE Transactions on Control Systems Technology, pp. 1-8, 2020.

[13] S. Wang, J. Kang, M. Degano, A. Galassini, and C. Gerada, "An accurate wide-speed range control method of IPMSM considering resistive voltage drop and magnetic saturation," IEEE Transactions on Industrial Electronics, vol. 67, pp. 26302641, apr 2020.

[14] T. Sun, M. Koc, and J. Wang, "MTPA control of IPMSM drives based on virtual signal injection considering machine parameter variations," IEEE Transactions on Industrial Electronics, vol. 65, pp. 6089-6098, aug 2018.

[15] J. Chen, J. Li, and R. Qu, "Maximum-torque-per-ampere and magnetization-state control of a variable-flux permanent magnet machine," IEEE Transactions on Industrial Electronics, vol. 65, pp. 1158-1169, feb 2018.

[16] K. Li and Y. Wang, "Maximum torque per ampere (MTPA) control for IPMSM drives based on a variable-equivalentparameter MTPA control law," IEEE Transactions on Power Electronics, vol. 34, pp. 7092-7102, jul 2019.

[17] E. Armando, R. I. Bojoi, P. Guglielmi, G. Pellegrino, and M. Pastorelli, "Experimental identification of the magnetic model of synchronous machines," IEEE Transactions on Industrial Applications, vol. 49, no. 5, pp. 2116-2125, 2013.

[18] S. Stipetic, J. Goss, D. Zarko, and M. Popescu, "Calculation of efficiency maps using a scalable saturated model of synchronous permanent magnet machines," IEEE Transactions on Industry Applications, vol. 54, pp. 4257-4267, sep 2018.

[19] C. Hackl, J. Kullick, and N. Monzen, "Optimale Betriebsführung für nichtlineare Synchronmaschinen," in Elektrische Antriebe - Regelung von Antriebssystemen (J. Böcker and G. Griepentrog, eds.), Springer-Verlag, 2020. 